\title{
Michael Brie
}

\section{Moskau: Das politische Regime der russischen Hauptstadt}

Mittlerweile haben sich in einer Reihe postsozialistischer Länder relativ stabile politische und wirtschaftliche Systeme herausgebildet. An die Stelle des Systemwandels ist der »normale« soziale Wandel innerhalb eines gegebenen Typus politisch-wirtschaftlicher Regime getreten. Die Analyse des Übergangs zu einem System, dessen wichtigste Charakteristika normativ vorgegeben sind, kann durch die Untersuchung des existierenden Systems politisch-wirtschaftlichen Austauschs, der Identifikation der wichtigsten Akteure dieses Austauschsystems und ihrer dominanten Interaktionsmuster sowie der weiteren evolutionären Tendenzen ergänzt werden. Gegenüber dem Vergleich mit den Indikatoren einer idealen Demokratie oder Marktwirtschaft wird der Vergleich mit Regimen interessant, die sich als unmittelbares Resultat ähnlicher politisch-wirtschaftlicher Wandlungsprozesses an anderem Ort und zu anderer Zeit herausbildeten.

Im folgenden werden die Genesis des gegenwärtigen politischen Regimes Moskaus, seiner wichtigsten Merkmale sowie absehbare Perspektiven seiner weiteren Evolution dargestellt. Die Konzentration auf ein einzelnes lokales Regime soll es erlauben, Prozesse, die auf nationaler Ebene oftmals zu komplex und unübersichtlich sind, in einer einfacheren Gestalt zu untersuchen. Dies scheint auch deshalb von Bedeutung, weil die politische Entwicklung der Stadt Moskau eine Reihe von Entwicklungen in den anderen Regionen Rußlands sowie innerhalb des föderalen politischen Systems antizipierte. (1) Nach einer kurzen Information über den politischen Regimewechsel in Moskau werden (2) das politische Rückgrat dieses neuen Regimes - die Staatsapparatepartei, (3) das sozioökonomische System dieses Regimes - ein munizipaler Kapitalismus, (4) das Verhältnis zwischen lokaler und föderaler Macht, (5) die Verbindung politischer und wirtschaftlicher Machtgruppen in Moskau dargestellt und (6) auf die Rolle von organisierter Kriminalität und Korruption eingegangen. Abschließend werden (7) einige Perspektiven des politischen Regimes in Moskau erörtert. Dazu wird auf das Konzept »urbaner Maschinen«, wie es für die nordame- 
rikanischen Großstädte des ausgehenden 19. Jahrhunderts entwickelt und seitdem auf weitere Länder angewendet wurde, zurückgegriffen.'

\section{Rur Entstehumg des gegenwärtigen politischen Regimes in Moskau}

Moskau ist seit 1918 die Hauptstadt zunächst der Sowjetunion und später der russischen Föderation. Seitdem war es das exklusive Zentrum der politischen, wirtschaftlichen und militärischen Macht. Hier wurden die wichtigsten wissenschaftlichen und kulturellen Institutionen zentralisiert und die Kontakte mit der Außenwelt kontrolliert. Zwischen 1900 und 1990 erhöhte sich die Zahl der Einwohner von einer Million auf mehr als neun Millionen. Moskau ist damit die bevölkerungsreichste und politisch wie wirtschaftlich einflußreichste Region des Landes. Bis zur Perestroika bildete das Moskauer Parteikomitee, das seit den zwanziger Jahren unter der direkten Kontrolle des Generalsekretärs der KPdSU stand, das lokale Machtzentrum.

Kurz nach seiner Emennung zum neuen Generalsekretär der KPdSU im März 1985 setzte Gorbatschow den Parteisekretär des Gebietes Sverdlovsk, Boris Jelzin, als Chef des Moskauer Parteikomitees ein. Ein sehr schneller und radikaler Austausch von Kadern erfolgte. Ein bedeutender Teil der exekutiven Macht wurde vom Parteikomitee in die neu von Industriemanagern besetzte Moskauer Stadtadministration verlagert. Die 1987 erfolgte Ablösung Jelzins konnte diesen Prozeß der Auflösung der Strukturen der Parteiherrschaft nicht mehr rückgängig machen. Die Welle der Liberalisierung und dann der Demokratisierung fand gerade in der Moskauer staatssozialistischen Mittelklasse ihren wichtigsten Rückhalt.

Im Gefolge der gleichzeitig mit den in der russischen Republik abgehaltenen Wahlen zu den örtlichen Sowjets wurde die lokale politische Macht in Moskau von einer Legislative übernommen, die durch die radikale Reformbewegung Demokratisches Rußland geführt wurde. Gavriil Popov, einer der bekanntesten Reformintellektuellen und radikaler Verfechter der Beseitigung der Herrschaft der kommunistischen Partei und der Durchführung markt-

1 Die wichtigsten Quellen der folgenden Analyse sind russische Zeitungen. Auch die umfangreiche und fundierte Darstellung des sowjetischen Moskaus und der ersten Jahre des postsozialistischen Wandels durch Timothy J. Coltons Buch rMoscow. Governing the Socialist Metropolis« (Colton 1995) konnte in wichtigen Fragen keine anderen Quellen erschließen. Zumeist sind die in den Artikeln gegebenen Informationen über Hintergrundzusammenhänge nicht überprüfbar. Der Moskauer Journalist Andrej Fadin bemerkt in diesem Zusammenhang, daß der relativ hohe Grad an Meinungsfreiheit in Moskau, wo es möglich ist, fast unangefochten Jelzin, Tschemomyrdin oder sogar die Paten der Unterwelt zu kritisieren, nicht gilt, wenn es sich um Stories handelt, die sich auf Moskauer Immobilien oder die Umverteilung von Eigentum in der Stadt beziehen. Keine andere Person sei derart mit einem Tabu belegt, was kritische Berichterstattung betrifft, wie der Moskauer Bürgermeister (Fadin 1996). Neben der genannten Arbeit von Colton sei auf die Artikel von Chinayeva (1996) und Razuvaev (1996) verwiesen, die erste Ansätze einer wissenschaftlichen Analyse des politischen Regimes in Moskau darstellen. 
wirtschaftlicher Reformen unter Nutzung autoritärer Mittel wurde Vorsitzender des Moskauer Sowjets. Jurij Luschkov, bis dahin der zweite Mann der Moskauer Stadtadministration, wurde zum neuen Chef der Exekutive. Ein Jahr später, parallel zu den ersten russischen Präsidentschaftswahlen, fanden Direktwahlen für das Amt des Moskauer Bürgermeisters und seines Stellvertreters statt. Popov und Luschkov erhielten rd. 67\% der abgegebenen Stimmen. Der Weg zu einem »präsidialen« System der Machtausübung innerhalb der Stadt Moskau war geöffnet worden. Nachdem Popov im Juni 1992 (nach mehreren folgenlosen Ankündigungen) zurücktrat, wurde Luschkov durch Jelzin zu dessen Nachfolger ernannt.

Im Mai 1993 wurde eine neue städtische Legislative, die Moskauer Duma, mit nur 35, dafür aber von der Ausübung anderer Funktionen befreiter Abgeordneten gewählt. Diese Wahlen signalisierten eine starke Unzufriedenheit, da mehr als 30\% der Wähler gegen alle aufgestellten Kandidaten stimmten und die Bewegung Vybor Rossii, die am Ende über eine knappe Mehrheit verfügte und die Moskauer Regierung unterstützte, nur knapp $20 \%$ der Wähler hinter sich hatte. Die turnusmäßig für 1995 festgelegten Neuwahlen zur Duma wurden auf 1997 vertagt. Im Juni 1996, wiederum parallel zu den russischen Präsidentschaftswahlen, kam es zu einer erneuten Bürgermeisterwahl, die Luschkov unangefochten mit $89 \%$ der Stimmen für sich entschied. Diese Ergebnisse zeigen, daß ein politischer Mechanismus entstanden war, der dem Bürgermeister (aber nicht der Legislative) breite politische Unterstützung der Bevölkerung und eine sichere Wiederwahl garantierte. Moskau wurde nach Jahren der Unruhe politisch zu einer relativ stabilen Stadt. Im folgenden wird versucht, die Entstehung dieses Mechanismus und seine wichtigsten Merkmale zu rekonstruieren.

Das neue politische Regime Moskaus entstand nicht in einem Vakuum, sondern in einer spezifischen institutionellen Umwelt, geprägt vor allem durch die Veränderungen auf der föderalen Ebene, und konfrontiert mit den Erwartungen und Gewohnheiten einer in vielen Jahrzehnten entstandenen politischen Kultur. Diese institutionelle Umwelt und politische Kultur ist geprägt durch: die Konzentration der politischen Auseinandersetzung auf einen Wettbewerb unterschiedlicher politischer Institutionen und informeller Einflußgruppen an der Stelle von Parteien und Organisationen; die fast ausschließliche Fixierung der Erwartungen der Bevölkerung auf die Exekutive; die Personalisierung sozialen Vertrauens; die hohe personelle Kontinuität der Moskauer Exekutive seit 1986 und die Mischung von individualistischen Strategien der Bewältigung der Umbruchsfolgen durch die Bevölkerung und der gleichzeitig bestehenden hohen Erwartungen an den Staat als Garant sozialer Sicherheit im weitesten Sinne (vgl. ausführlich dazu Brie 1996a und 1997a). 


\section{Das institutionelle Rückgrat des neuen Regimes - die Staatsapparatepartei}

Vor dem Hintergrund der gegebenen institutionellen Umwelt und politischen Kultur des postsowjetischen Rußlands suchten lokale politische Unternehmer Strategien zu formulieren, die ihnen gegenüber ihren Konkurrenten einen Vorsprung im offen entbrannten politischen Wettbewerb zu sichern schienen. Es setzten sich jene durch, die in der Lage waren, die institutionellen Strukturen und die Verteilung der Ressourcen so zu beeinflussen, daß die Formulierung von Alternativen blockiert wurde, während zugleich die eigenen Positionen ausgebaut werden konnten. Die Entstehung des gegenwärtigen politischen Regimes von Moskau war weder die Implementation eines fertigen Plans, noch das Ergebnis eines völlig spontanen Prozesses. Die Moskauer Regierung unter Führung zunächst von Popov und dann von Luschkov war ganz offensichtlich der dominante lokale Akteur des Regimewandels. Sie war in den meisten Fälle dazu in der Lage, eine den aktuellen Problemen und den eigenen Interessenlagen angepaßte gradualistische Strategie durchzusetzen, die einige dauerhafte Ziele sowie eine eher inkrementelle, Versuch und Irrtum einkalkulierende Weise der Umsetzung einschloß.

Drei der wichtigsten Elemente dieser Strategie der Moskauer Regierung sollen hier hervorgehoben werden. Erstens: Zu einer Zeit, als die Mehrheit der anderen Regionen durch die Dominanz der neu gewählten Legislative, der örtlichen Sowjets, geprägt waren, führte die Allianz aus autoritären Radikalreformern um Popov und der aus der sowjetischen Industrie kommenden städtischen Manager um Luschkov das schon erwähnte System der Direktwahl des Bürgermeisters ein. Beide Gruppen waren an einer städtischen Regierung mit hoher Machtkompetenz und Legitimation interessiert - erstere in der Hoffnung, dadurch die Einflüsse »konservativer« staatssozialistischer Interessen abwehren und möglichen Massenprotesten entschieden begegnen zu können, letztere im Bestreben, den Transformationsprozeß unter der ständigen und detaillierten Kontrolle der städtischen Administration zu halten.

Zweitens: Im Bestreben, die anfangs durchaus gegebene Vetomacht des Moskauer Sowjets einzuschränken, wurde ein kompliziertes System von Beratungs- und Kontrollmechanismen geschaffen, wodurch Entscheidungsprozesse unübersichtlich gestaltet wurden. In der Realität stärkte diese Inflation »partizipatorischer« Institutionen die städtische Exekutive (Davydova 1991). Sie wurde dadurch zum einzigen Ort, der überhaupt bindende Entscheidungen, und sei es per Dekret an allen anderen Institutionen vorbei, fällen und implementieren konnte. Die 1993 erfolgte Reduktion der städtischen Duma auf 35 Abgeordnete hat die Legislative angesichts der Schwäche der Parteien in die Abhängigkeit von der Exekutive getrieben, die den Abgeordneten im Austausch gegen Loyalität einen be- 
stimmten Einfluß auf konkrete Entscheidungen sowie den Ausbau klientelistischer Netzwerke und damit Chancen für eine Wiederwahl sicherte.

Drittens: Im Rahmen der Zentralisation von Entscheidungsmacht und Ressourcen wurde die territoriale Gliederung der Stadt verändert. Zu sowjetischen Zeiten gab es 33 relativ große Stadtbezirke (rajony), die unter der direkten Kontrolle des Moskauer Parteikomitees standen. Popov und Luschkov führten ein System von zehn Distrikten ein, deren Chefs, die Präfekten, direkt durch den Bürgermeister eingesetzt werden. Jeder der administrativen Distrikte besteht aus rd. 15 Mikrobezirken (mikrorajony), die jeweils 60 bis 70 Tausend Einwohner haben. Dieses dreistufige System soll eine strikt hierarchische Pyramide bilden, in der ein System direkter persönlicher Verantwortlichkeit von unten nach oben durchgesetzt wird.

Diese Veränderungen in der institutionellen Struktur etablierten ein Regime, an dessen Spitze ein direkt gewählter Bürgermeister steht, der - einmal gewählt - fast völlig unabhängig von der Legislative, Judikative und von Parteien handeln kann. Die gesamte Exekutive ist ausschließlich ihm unterstellt. Die Gesetzgebung befindet sich gleichfalls weitgehend unter seiner persönlichen Kontrolle. Die Gerichte sind durch die extreme Widersprüchlichkeit der föderalen und der lokalen Gesetze paralysiert. Im Zweifelsfall werden Entscheidungen durch Beschlu $\beta$ der Exekutive auch dann herbeigeführt, wenn sie die Gesetzeslage eindeutig verletzen. Es handelt sich um ein politisches Regime personaler, durch Direktwahlen prozedural legitimierter Herrschaft des Bürgermeisters.

Diese Herrschaft ist weder in ein System von Parteien eingebunden, die ihre staatlichen Repräsentanten zu kontrollieren vermöchten, noch ist sie bisher zu einer bloßen Branche der Macht des russischen Präsidenten herabgesunken. Führende Repräsentanten der zunächst durch das Moskauer Komitee der Kommunistischen Partei und dann durch den demokratisch gewählten Moskauer Sowjet eingesetzten und bestätigten Exekutive vermochten es, die städtische Administration schrittweise in einen Apparat zu verwandeln, der den Transformationsprozeß der russischen Hauptstadt auf das engste mit der eigenen Machterhaltung verbindet. Nachdem eine solche Symbiose erreicht war, stellte sich der Chef der Exekutive der erneuten Direktwahl. Die Regierungsorgane werden unmittelbar in ein Instrument zur Erzielung von Wahlerfolgen ihrer Führer verwandelt - in Glieder einer Staatsapparatepartei. ${ }^{2}$ Die Loyalität der Beamten gilt in diesem Fall nicht primär dem Amt, sondern dem direkten oder mittelbaren Vorgesetzten. Bei aller strukturellen Ähnlichkeit und Kontinuität gegenüber der kommunisti-

2 Der Terminus »Staatsapparatepartei« wurde gewählt, um den russischen Begriff der partija vlasti zu übersetzen. Vlasti' steht in der russischen Sprache a) für die Macht allgemein, b) für die Organe der politischen Machtausübung und administrativen Leitung sowie c) für Personen, die staatliche Macht ausüben (Ožegov 1987: 71). 
schen Staatspartei (Kolosov 1995: 23) ist unter den Bedingungen der Direktwahl der Chefs der Exekutive das wichtigste Erfolgskriterium verändert - nicht Emennung von oben, sondern der persönliche Wahlerfolg.

Einer solchen »Staatsapparateparteik fehlt das elementarste Merkmal einer Partei - sie ist keine auf (formal) freier Werbung beruhende Vergesellschaftung, die vom Staat verschieden ist (Weber 1976: 167). Nicht der Staat wird durch außerstaatlich konstituierte und miteinander konkurrierende Parteien erobert, um dessen Positionen zu besetzen, sondern der prädemokratisch besetzte Staat wird genutzt, um diese Positionen zu verteidigen und auszubauen. Außerstaatliche politische Organisationformen werden systematisch benachteiligt. Die Patronage der Bevölkerung durch die Regierung wird zur wichtigsten Form der Präsentation öffentlicher Güter. Die Stärke einer solchen führergeleiteten Staatsapparatepartei liegt in ihrer Fähigkeit, die staatlichen Ressourcen fast unkontrolliert für die eigenen Interessen einsetzen zu können. Die Moskauer Regierung kann die Arbeit der gesamten städtischen Administration in den Dienst einer Wahlkampagne stellen, das Budget entsprechend umverteilen und einflußreiche Personen oder Gruppen durch Formen der individuellen Patronage bzw. der Patronage großer Gruppen durch städtische Verwaltungseinrichtungen (Massenpatronage) zu binden suchen. Dies sind außerordentlich starke Waffen im politischen Wettbewerb, wenn der Gegner nichts anderes vermag, als Frustration zu mobilisieren. Wahlen nehmen die Form von Alles-oderNichts-Spielen an, wo der Gewinner in den Genuß der kompletten Kontrolle über den Staat gelangt, und der Verlierer ohne jeden politischen Einfluß bleibt. Innerhalb des institutionellen Systems gibt es keinen Platz für eine einflußreiche Opposition, da die Legislative schwach ist.

\section{Moskaus munizipaler Kapitalismus}

Der bekannteste Konflikt zwischen der föderalen Regierung Rußlands und der Moskauer Stadtregierung betraf die Privatisierungsstrategie. Die Reformer um Gajdar strebten eine möglichst rasche Privatisierung an, um die Irreversibilität der Reformen zu sichern und nahmen dafür die ökonomische Ineffizienzen der neuen Eigentumsformen in Kauf. Anstatt die anfangs erklärten Ziele der Trennung zwischen der Kontrolle durch Eigentümer und der Ausübung des laufenden Managements sowie einer breiten Streuung der Aktien zu verfolgen, erkauften die Reformer die Unterstützung der Privatisierung (oder doch zumindest deren Duldung) durch die einflußreichen Direktoren der staatlichen Großbetriebe damit, daß sie es zuließen, daß bei der ersten Privatisierungswelle 1992/93 die Insider-Privatisierung dominierte (Sutela 1994: 430). Sich ihrer Stellung in Moskau sicher, war die aktive politische Erschaffung einer Infrastruktur des regulierten Marktes und die soziale Stabilisierung 
dieses Prozesses das vordringliche Ziel der Moskauer Regierung und der Hauptweg eigener Machtsicherung. In der öffentlichen Auseinandersetzung mit führenden russischen Reformern führte Luschkov aus:

"Eine zivilisierte Wirtschaft entsteht nicht als Resultat spontanen Wachstums. Sie wird geschaffen durch Detailarbeit, durch eine Regulation ihrer Mechanismen, die die konkreten Bedingungen, die spezielle lokale Umwelt und die Gewohnheiten der Menschen beachtet. Sie sprießt nicht wie Unkraut auf brachem Land. Systeme stellen eine Art lebendigen Organismus dar. Um sie mit Geist zu beseelen, muß man arbeiten wie Gott in den Tagen der Schöpfung « (Luschkov 1996a: 159)

Der sowjetische Sozialtechnokratismus wird in einen postsowjetischen Reformtechnokratismus übersetzt. Im Konkreten setzt sich diese Reformstrategie aus drei Komponenten zusammen - (1) einem speziellen Weg der Privatisierung, (2) der zentralen Rolle des munizipalen Eigentums und (3) einer starken sozialen und nationalen Orientierung der Reform. Das politisch wichtigste Produkt einer solchen Strategie ist ein solcher Umbau der wirtschaftlichen Strukturen, der die Mehrheit der Bevölkerung und bedeutende Segmente der neuen Unternehmerschaft in deutlicher Abhängigkeit von der Patronage durch die Moskauer Regierung beläßt und zu politischer Loyalität und Wahlunterstützung bewegen soll.

\section{Die Moskauer Privatisierungsstrategie}

Die Moskauer Regierung wandte sich von Anfang an entschieden gegen die Privatisierungspolitik der russischen Regierung. Es kam zu einer Konfrontation zwischen beiden, die erst durch direktes Eingreifen des Präsidenten gelöst wurde. ${ }^{3}$ Durch Dekrete, die gleichermaßen am Obersten Sowjet Rußlands und der russischen Regierung vorbei durch Jelzin erlassen wurden, erhielt Moskau das Recht, einen eigenen Weg der Privatisierung zu beschreiten. Die Gründe, die die Moskauer Regierung vorbrachte, waren vordergründig nicht ideologisch, sondern pragmatisch. Angesicht der wirtschaftlichen Krise und der sich zuspitzenden sozialen Probleme blieben der Stadtverwaltung nur drei wichtige Ressourcen - der städtische Immobilienbesitz, die städtischen Anteile an Unternehmen und Banken sowie föderale Subventionen. In einer Situation, wo es fast unmöglich war, wirkungsvoll Steuern zu erheben (sowohl aufgrund der wirtschaftlichen Lage wie des fast völlig fehlenden Systems der Steuererhebung) wurde der öffentliche Immobilienbesitz zur wichtigsten Einnahmequelle und zum bedeutendsten Instrument sozioökonomischer Steuerung.

3 Luschkov gibt einen Einblick in den Stil russischer Politik, wenn er beschreibt, wie die Entscheidung Jelzins zustande kam. Luschkov habe sich an diesen telefonisch gewandt, als der damalige Moskauer Bürgermeister, Popov, unter Verweis auf den Widerstand der Zentralregierung sowie des Moskauer Sowjets, mit Rücktritt drohte. Jelzin habe die Moskauer Regierung daraufhin am folgenden Tage empfangen und der Stadt einen Sonderstatus erteilt (Luschkov 1996a: 198). 
Die Moskauer Regierung sah das öffentliche Eigentum an fast dem gesamten Immobilienbesitz - eine der wichtigsten Hinterlassenschaften der Sowjetunion - als zentralen Hebel der Wirtschaftsreformen an. Sie beschloß, in den ersten Jahren keinerlei Privatisierung des städtischen Immobilieneigentums zuzulassen. Dabei nutzte sie den Umstand, daß sich in der Stadt schon in der späten Perestrojka der Hauptanteil des neuen Banksektors, des privaten Großunternehmertums sowie der ausländischen Investitionen konzentrierte. Dies führte zu einem explosionsartigen Anstieg der Immobilienwerte. Beginnend mit dem Jahr 1992 wurde die Pacht zur Hauptform, um öffentlichen Immobilienbesitz in private Nutzung zu überführen. 1995 erlaubte die Moskauer Regierung, die Pachtrechte an Dritte weiterzuverkaufen und nahm dabei zusätzliche 1,5 Billionen Rubel ein. Während die föderale Regierung 1995 insgesamt vier bis fünf Billionen Rubel aus der Privatisierung (einschließlich des Öl- und Gassektors) einnahm, konnte die Moskauer Regierung alleine 800 Milliarden Rubel verbuchen (Michajlov 1996). Es entstand ein regulierter Markt für Pachtrechte. 1996 gründete die Stadt eine Immobilienbörse und tritt dabei als wichtigster Vermittler auf. Es gibt Überlegungen, das Steuersystem von der Gewinn- und Mehrwertsteuer auf die Besteuerung von Immobilien umzustellen, was Steuerhinterziehungen erschweren und zu einer effektiveren Nutzung der Immobilien führen soll. Gleichzeitig erhofft man sich dadurch einen Stimulus für Investitionen (Melničenko 1996). Die Moskauer Regierung versuchte, eine Regelung zu treffen, um alle Verträge über die Übertragung von Immobilien annullieren zu können, wenn die vereinbarte Summe unter dem Marktpreis zu liegen scheint, und sich selbst das Recht zu erteilen, die Immobilie zum vereinbarten Preis zu kaufen. Dies würde ihr ein faktisches Monopol auf den Kauf von Immobilien geben. Die Stadt nutzt das öffentliche Immobilieneigentum als Einlagekapital in privaten Unternehmen und profitierte stark vom Wertzuwachs der Immobilien in den letzten Jahren. Sie gründete eine eigene Organisation (Mosinterstroj), um private Investitionen in städtische Immobilien zu fördern. Zu einer Zeit, wo viele andere Regierungen in der Welt auf Privatisierung setzen, erhofft sich die Moskauer Regierung besondere Vorteile von gemischten Eigentumsformen (Artsruni 1996). Ihr Ziel ist es, die eigenen Einnahmen durch strikte Kontrolle der Finanztransaktionen zu erhöhen (AEN 1994), die Investitionen zu lenken und die neuen Unternehmen in die städtische Ökonomie zu integrieren. Sie hat dabei direkten Einfluß auf die Entwicklung des Verbrauchermarkts genommen (Babak 1993a; Efremova 1993) und eine Reihe von Verträgen mit ausländischen Firmen annulliert, die ihrer Ansicht nach den gegenüber der Stadt eingegangenen Verpflichtungen nicht nachgekommen sind (Babak 1993b). 
Die zentrale Rolle des munizipalen Eigentums

Im Gefolge der gewählten Privatisierungsstrategie ist die Moskauer Regierung zum wichtigsten Eigentümer und Unternehmer der städtischen Wirtschaft geworden. Sie hat die Kontrolle über das Immobilieneigentum behalten und als Kapitalanteile in privatisierten und neu gegründeten Unternehmen eingesetzt, eigene Unternehmen organisiert und ein System abhängiger Banken und Firmen geschaffen. Ein bedeutender Teil der städtischen Dienstleistungen ist formal privatisiert und an Subunternehmer weitergegeben worden. Die städtische Regierung besitzt 14\% des Einlagekapitals aller Unternehmen, die auf der Basis von früherem städtischen Eigentum gebildet wurden und hat ein eigenes Systems der Wirtschaftskontrolle geschaffen. Das in Bau befindliche hochmoderne Dienstleistungszentrum unterhalb des Manegeplatzes beim Kreml und das Hotel National befinden sich völlig im städtischen Besitz. $51 \%$ von Pizza Hut, $75 \%$ der Aktiengesellschaft Moskva, die die öffentlichen Anteile in Moskauer Hotels verwaltet, $67 \%$ der Gesellschaft AZS-Servis, die viele der Moskauer Tankstellen kontrolliert, 49\% von Moskaus McDonalds, 74\% einer Fernsehproduktionsgesellschaft und 25\% einer Fernsehgesellschaft sind (nach Daten von 1995) in munizipaler Hand (Razuvaev 1996: 42). Die Einkünfte aus diesen Firmen bilden nach denen aus dem Verkauf oder der Verpachtung von Immobilien die zweitwichtigste Einnahmequelle der Stadt.

1996 übernahm die Moskauer Regierung das bankrotte Autowerk ZIL. Die föderale Regierung wurde zu Staatsaufträgen für dieses Unternehmen gedrängt. Der Bürgermeister Luschkov ist bestrebt, diesen und eine Reihe anderer Großbetriebe unter staatliche Patronage zu stellen und ihren Umbau strikt zu kontrollieren. Er sieht darin den einzig möglichen Weg ihrer Rettung, solange privates Kapital und Unternehmertum dafür nicht gewonnen werden können, Auf der Suche nach zusätzlichen Einnahmen versuchte Luschkovs Regierung, die öffentlichen Finanzen gegen Zinsen als zeitweilige Kredite an private Banken zu vergeben. Im Konflikt mit der Föderationsbank und der föderalen Regierung wurde der Moskauer Haushalt seit Februar 1993 über ein System autorisierter Banken wie Most-Bank, Inkombank, Stoličnyj Bank und Menatep realisiert. Vladimir Gussinskij, damals enger persönlicher Vertrauter von Luschkov und Direktor der Most-Bank, wurde Chef des Komitees dieser Banken.

1994 wurden rd. 8 Mrd. Dollar vermittelt über das System dieser autorisierten Banken realisiert (Chinayeva 1996: 31). Die Moskauer Regierung nutzte den Wettbewerb der Banken um den Zugang zu diesen Ressourcen, um vorteilhafte Bedingungen durchzusetzen. Die Banken wurden gedrängt, als Patrone für soziale und kulturelle Projekte der Hauptstadt aufzutreten. In den ersten sechs Monaten desselben Jahres gaben die Banken rd. 5 Mrd. Rubel für solche Projekte aus und investierten eine halbe Mrd. Dollar in 
Moskaus Wirtschaft (Jasina 1994). Im März 1995 wurde eine eigene munizipale Bank Moskaus gegründet, um die Kapitalisierung des Haushalts unter die direktere Kontrolle der Regierung zu bringen (Ivanov 1996). Damit ist es zu einer weiteren Machtkonzentration gekommen. Zugleich wird eine höhere Unabhängigkeit von der föderalen Regierung angestrebt. Die Moskauer Stadtregierung hat sich weitgehend als vertrauenswürdiger Geschäftspartner erwiesen. Ihre Fähigkeit, günstige wirtschaftliche und soziopolitische Bedingungen zu schaffen sowie Moskaus Rolle als monopolistisches Zentrum für Politik und Finanzen hat dazu geführt, daß 44\% der 2,8 Mrd. Dollar Auslandsinvestitionen des Jahres 1995 allein in Moskau getätigt wurden. 350.000 kleine und mittlere Unternehmen wurden in den letzten Jahren gegründet. Dies und der große öffentliche Sektor sind die wichtigste Ursache für die geringe Arbeitslosenquote in Moskau.

\section{Die soziale und nationale Orientierung der Wirtschaftsreformen}

Die Politik der Moskauer Regierung unter Luschkov ist darauf gerichtet, einen Teil des neuen Reichtums zugunsten der sozialen Bedürfnisse der sozial schwachen Gruppen der Bevölkerung zu sozialisieren, die öffentliche Infrastruktur, die durch die Mehrheit der Einwohner genutzt wird, auszubauen, Erwartungen der alten und der neuen Mittelklasse zu erfüllen und nicht zuletzt jene Funktionen zu erfüllen, die »die Hauptstadt einer großen Nation« hat (Luschkov 1996d). Während seiner Wahlkampagne für den Posten des Bürgermeisters im Juni 1996 konnte Luschkov auf eine Reihe von Ergebnissen dieser Politik verweisen. Er schrieb: Die Wahlergebnisse werden zeigen,

»was die 16 Millionen Quadratmeter neuer Wohnfläche, die 60 Millionen Quadratmeter reparierter Straßen, Brücken und Tunnel, die Wiederentstehung des historischen Zentrums der Hauptstadt mit ihren Heiligtümern und kulturellen Sehenswürdigkeiten, die Zuzahlungen für Rentner, die Gewährleistung sozialer Sicherheit für Gruppen mit geringem Einkommen, die freie Fahrt mit den Transportmitteln des öffentlichen Verkehrs usw, in den Augen der Bevölkerung wert sind « (Luschkov 1996d).

Die sozialen Leistungen der Moskauer Regierung sind vor dem Hintergrund der schweren wirtschaftlichen Probleme durchaus beeindruckend. Die sowjetische Politik der Sicherung von Grundbedürfnissen für alle wurde weitgehend fortgesetzt. Über die genannten sozialen Maßnahmen hinaus zahlt die Moskauer Regierung den öffentlichen Angestellten 40\% über Tarif, um die höheren Ausgaben in Moskau abzudecken, sichert die regelmäßige Zahlung der Rente, finanziert den sozialen Wohnungsbau mit Gewinnen aus dem kommerziellen Wohnungsbau und trägt $60 \%$ der anfallenden Wohnkosten der Bevölkerung. Allein für letzteres werden $43 \%$ des städtischen Haushalts verausgabt (Nemcov 1997).

Eines der drängendsten Probleme Moskaus ist der Verkehr. Die Regierung setzt den Ausbau der Metro fort, Moskaus wichtigstem öffentlichen Trans- 
portsystem. Konfrontiert mit einer Explosion des Autoverkehrs, konzentriert sich die Regierung auf den Ausbau des Straßensystems. Der Straßenring um Moskau wird zu einer modernen Autobahn ausgebaut. Zugleich entsteht ein innerer Autobahnring (Presnjakova 1996). Parkhäuser für eine halbe Million Autos sollen in den nächsten Jahren gebaut werden, um ein Fiasko in einer Zehnmillionen-Stadt zu beheben, die faktisch nur für den öffentlichen Nahverkehr gebaut wurde (PriMERnaja programa 1996).

1991 begann die Rekonstruktion des historischen Zentrums Moskaus. Es ist Luschkovs ehrgeizigstes Projekt, Moskau sein historisches Gesicht, das im Gefolge des Stalinschen Plans des städtischen Umbaus zerstört wurde, zurückzugeben und es zugleich in eine moderne Metropole zu verwandeln. Die Architektur, die unter direkter Anleitung und persönlicher Kontrolle Luschkovs entsteht, zeichnet sich durch eine Mischung aus vorrevolutionärem Neotraditionalismus und extremer Modernität aus und drückt ein Verständnis von Rußland als traditionaler Großmacht mit eigener Zivilisation aus, die den Anspruch erhebt, eine der führenden Nationen des 21. Jahrhunderts zu werden.

Die Moskauer Regierung vertritt auch eigene außenpolitische Akzente. Aus dem städtischen Haushalt werden russische Truppen in Tadjikistan und der Bau von U-Booten unterstützt (Kasjanikova 1996). Luschkov ist Vorsitzender der Moskau-Sewastopol-Stiftung, die Sewastopol, auf der Krim gelegen und gegenwärtig Teil der Ukraine, für Rußland reklamiert (Luschkov 1996c). Harte und zum Teil illegale Aktionen wurden gegen Bürger anderer GUS-Staaten sowie von autonomen Republiken Rußlands unternommen, die für die steigende Kriminalität verantwortlich gemacht werden (Puškar 1994; Moijseev/Uchlin 1994).

In den letzten sieben Jahren wurde das sozioökonomische System Moskaus aus einem von der imperialen Revenue der Sowjetunion profitierenden und gleichwohl sehr beschränkten Wohlstandssozialismus in das eines munizipalen Kapitalismus transformiert, der seine Revenue aus den gleichfalls beschränkten Gewinnen der gesamtrussischen Transformation zieht und deshalb auch nicht (oder doch zumindest nicht in den nächsten Jahren) zu verallgemeinern ist. In enger Kooperation mit wichtigen Unternehmergruppen ist die Moskauer Regierung zu einem der dynamischsten Unternehmer der Stadt aufgestiegen. Sie kontrolliert die zentralen ökonomischen Prozesse der Stadt, verfügt über die wertvollsten Immobilien und die größten Finanzsummen. Im Unterschied zum Großunternehmertum, daß mit einer Vielzahl von staatlichen Agenturen und ausländischen Unternehmen verhandelt, ist das kleine Unternehmertum und die große Masse der Bevölkerung weitgehend von der Massenpatronage innerhalb dieses munizipalen Kapitalismus abhängig. Dieses System ist gegenüber dem des Staatssozialismus durch weit größere soziale Ungleichheit gekennzeichnet, 
gibt den einzelnen mehr Wahfreiheit und zwingt sie zu größeren Eigenanstrengungen. Aber auch weiterhin stellen staatliche Patronage und Abhängigkeit der Bevölkerung von den konkreten Sozialleistungen der Regierung ein Hauptmerkmal des sozioökonomischen Systems dar.

\section{Verbündeter und Rivale - der Moskauer Bürgermeister in der föderalen Machistruktur}

An der feierlichen Inauguration Luschkovs als direkt gewählter Moskauer Bürgermeister im Juni 1996 nahmen nicht nur der russische Regierungschef Tschernomyrdin und eine Reihe weiterer Führungspersonen der russischen Politik teil, sondern auch der russische Präsident, Boris Jelzin, und der Patriarch Rußlands, Aleksij II. (Davydova/Zagrebnoj 1996). Dies ist ein deutlicher Indikator für Luschkovs herausragende Stellung. Seit Jahren zählen Experten ihn unverändert zu den fünf einflußreichsten Politikern Rußlands. Die häufigen Wechsel im inneren Zirkel der russischen Elite haben ihn bisher nicht berührt.

Diese stabile Position Luschkovs ist vor allem seiner Zugehörigkeit zu einer speziellen Gruppe russischer Politiker geschuldet. Im Unterschied zu jenen, die ihren zeitweisen Einfluß vor allem ihrer Stellung im persönlichen Stab des Präsidenten verdanken, verfügt Luschkov genauso wie Tschernomyrdin über eine eigene Machtbasis, die ihm nicht ohne negative Folgen für die föderale Elite entzogen werden kann - in seinem Fall die demokratisch legitimierte Regierungsgewalt über die Stadt Moskau. Zur Stärkung seiner Position hat Luschkov die Direktkontakte zwischen Moskau und einer größeren Zahl russischer Regionen ausgebaut und dabei Funktionen übernommen, die nach der Gesetzeslage nur föderalen Organen zukommen.

Die Ereignisse der letzten Jahre zeigten, daß Luschkov in der Lage ist, gegenüber einzelnen politischen Gruppen der föderalen Elite erfolgreich zu bestehen. Er hat den Kampf gegen Tschubais um das Privatisierungsprogramm gewonnen und war fähig, sich gegen die Angriffe des früheren Sicherheitschefs Jelzins, Korschakow, zu behaupten. 1994 machten die föderalen Subventionen 15,3\% des Moskauer Haushalts aus. Unter den Bedingungen einer schweren Finanzkrise konnte Luschkov über $90 \%$ jener Mittel aus dem föderalen Haushalt erhalten, die er beanspruchte (Kommersant Daily, 16. März 1995, S.3). In kritischen Situationen vermochte er sich immer wieder der persönlichen Unterstützung Jelzins zu versichern und garantierte diesem seine eigene Loyalität. Ohne Zögern unterstützte er 1991 und 1993 Jelzin und trug wesentlich zur politischen Stabilität in Moskau bei, einer kritischen Variable für alle russischen Machthaber. Luschkovs Kritik an der Regierungspolitik wurde niemals auf eine Kritik an Jelzin ausgeweitet.

Das Bündnis zwischen Jelzin und Luschkov war und ist aber ein sehr ungleiches. Die Angriffe gegen letzteren, die sich um die Jahreswende 1994/95 aus 
der Umgebung des Präsidenten häuften, sollten nicht nur Luschkovs Einfluß reduzieren, sondern vor allem ausschließen, daß er 1996 als Gegenkandidat zu Jelzin antreten könnte (Chinayeva 1996: 32f). Beamte, die Luschkov direkt unterstelit waren, wurden abgelöst und die eng mit ihm verbundene Most-Bank durchsucht. Luschkov erklärte öffentlich, daß er bereit wäre, auf Wunsch des Präsidenten zurückzutreten. Dieser wartete ein halbes Jahr, bevor er sich vor Luschkov stellte - als klar wurde, daß Luschkov bereit war, einen bestimmten Einfluß anderer föderaler Gruppen in Moskau zu dulden und Ambitionen auf das russische Präsidentenamt zunächst zurückzustellen. Nach der Präsidentenwahl von 1996 scheint sich Luschkov auf eine neue Runde des Kampfes um die Macht auf höchster Ebene vorzubereiten. Er äuBert sich immer aktiver zu Fragen der nationalen und internationalen Politik. Autoritäre und nationalistische Motive werden stärker. Aktiv sucht er ein Bündnis mit den Nachfolgem der kommunistischen Gewerkschaften und vertritt ein korporatistisches Modell für Rußland (Želenin 1997). Moskau wird als Modell für Gesamtrußland dargestellt. Solange aber Jelzin nicht abtritt $^{4}$, wird Luschkov Juniorpartner bleiben und versuchen, seine Position innerhalb der föderalen Elite und der Öffentlichkeit auszubauen, ohne dabei die Mehrheit der Eliten geschlossen gegen sich aufzubringen.

\section{Die »Moskauer Gruppe«}

Wie die Wahlen zum Posten des Bürgermeisters im Jahre 1996 zeigten, ist es Luschkov gelungen, die Entstehung politischer Alternativen zu seiner Person und der von ihm repräsentierten Politik völlig zu blockieren. Dies setzt voraus, daß alle einflußreichen politischen und wirtschaftlichen Gruppen mehr an der Erhaltung des etablierten Status quo als an der (aufwendigen) Formierung einer Alternative interessiert sind. Er scheint ihnen zumindest die Erzielung eines nicht allzu geringen gegenwärtigen Nutzens zu garantieren, während Versuche der Veränderung (und nicht genau abschätzbarer Nutzenmaximierung) von der Gefahr überschattet sind, auch dieses Nutzens verlustig zu gehen.

Vor den schon erwähnten Attacken von Mitgliedern des Apparats des russischen Präsidenten 1994/95 schien es vielen Beobachtern unstrittig, daß Luschkov der autoritative Führer einer weitgehend geschlossenen Gruppe politischer und wirtschaftlicher Organisationen sei, die über ein eigenes Sicherheitssystem verfüge. Einer der einflußreichsten Analytiker russischer Interessengruppen, Vladimir Lepechin, selbst Politiker und Unternehmer, räumte noch 1995 der Moskauer Gruppe Luschkovs eine stärkere Macht ein als der des Präsidenten:

4 Nach geltender Verfassung, die wohl ohne einen De-facto-Staatsstreich in diesem Punkt nicht geändert werden kann, darf Jelzin nicht ein drittes Mal kandidieren. 
"Diese Gruppe verfügt über ein klares Bewußtsein ihrer Interessen und eine entwickelte korporatistische Idee, riesige materielle und finanzielle Ressourcen, gute Kontakte zu allen föderalen Strukturen und Führern ..., solide autonome Sicherheitskräfte, eine große Gruppe von Massenmedien steht mehr oder minder unter ihrer Kontrolle ..., ebenso viele politische Führer und Beamte ...« (Lepechin 1995: 34).

Er ignorierte die Fähigkeit Jelzins, im Krisenfall die gesamte Elite unter seiner Führung zu einen und Kontrahenten rigoros auszuschalten. Jelzin verfügt über die wichtigste politische Ressource Rußlands - die Institution der Präsidentschaft, seit Oktober 1993 das alternativlose Zentrum der politischen Legitimation in Rußland.

Während der Angriffe auf ihn und seine Verbündeten konnte Lužkov 1994/95 keine der genannten Ressourcen wirkungsvoll ausspielen. Dies ist zwar kein Zeichen dafür, daß es keinerlei »Moskauer Gruppe« gibt, wie Razuvaev meint (1996: 41), aber es verweist auf die engen Grenzen, die ihren gemeinsamen Interessen und der Fähigkeit gezogen sind, diese im Konfliktfall föderal organisiert geltend zu machen. Mit Ausnahme von Vladimir Gussinskij, der direkt durch den Präsidialapparat angegriffen wurde, hat keiner der Unternehmer und Bankiers öffentlich Partei ergriffen. Es gab auch keinerlei Gedanken daran, etwa zu bewaffneten Mitteln zu greifen.

Unter anderen Bedingungen und bezogen auf andere Konflikte ist es Luschkov aber durchaus gelungen, charismatische Qualitäten zur Geltung zu bringen und sein enges Netzwerk einflußreicher Beamter, Geschäftsleute und Politiker zur Wahrung eigener und regionaler Interessen zu nutzen. Dies geschieht jedoch unterhalb der Ebene einer möglichen Auseinandersetzung mit dem russischen Präsidenten. Die Moskauer Gruppe stellt eine Art von Koalition partiell interdependenter Akteure dar, ein System des Austauschs von Leistungen im wechselseitigen Interesse und der Artikulation sowie Repräsentation einer Reihe gemeinsamer Anliegen unter der Führung von Luschkov. Dies schließt weder totale Subordination noch eine Bindung auf Gedeih und Verderb ein (vgl. Figur 1).

Eine detaillierte Analyse der wichtigsten Einflußgruppen Rußlands durch Sergej Baranov kommt gegenüber Lepechin zu einer weit differenzierten Einschätzung der realen Rolle der Moskauer Gruppe. Unter Nutzung der Daten von 1992 bis 1995 wird sie nach der Administration des Präsidenten, dem Sicherheitsrat und der Zentralbank als viertstärkste Gruppe dieser Jahre eingeordnet. Die Moskauer Gruppe bestehe einerseits aus der Moskauer Regierung als einem strikt hierarchischen $»$ Konzern « und andererseits einem $»$ Syndikat «, das durch die horizontale Symbiose der Moskauer Regierung und einiger Führungspersonen wichtiger Moskauer Banken und Unternehmen gebildet wird (Baranov 1996: 9). Die Moskauer Gruppe repräsentiert auf der einen Seite spezielle regionale Interessen der Hauptstadt 
Figur 1: Ein Modell des Akteursystems des politischen Regimes in Moskau

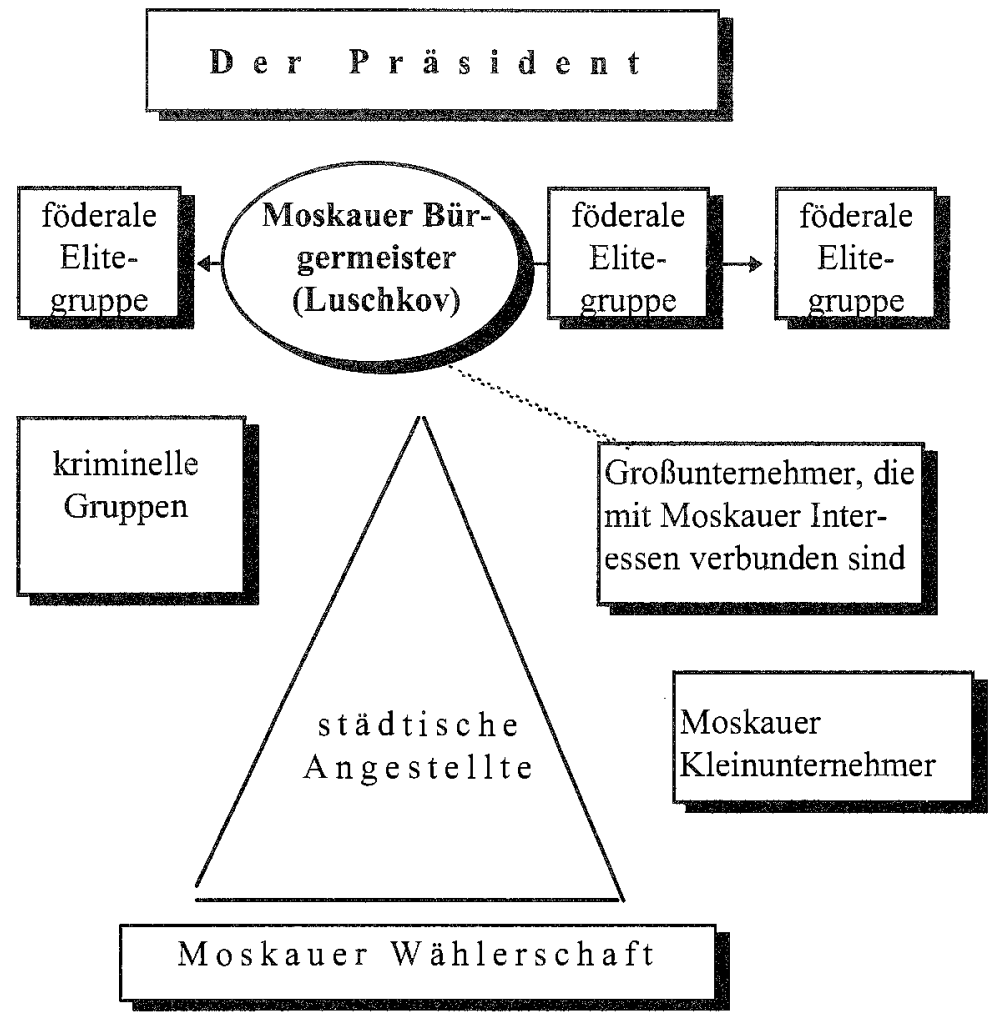

und ein Modell politischer und wirtschaftlicher Reformen, die sich als Vertretung dieser Interessen darstellen, und auf der anderen Seite die Interessen vor allem jener Gruppen aus Hochfinanz und Industrie, die sich gegen die Dominanz des Erdöl- und Erdgassektors richten und auf einen »nationalen Kapitalismus« mit einem starken Binnenmarkt orientiert sind. In den letzten zwei Jahren hat Luschkov seine Versuche intensiviert, die direkt durch ihn kontrollierten Machtressourcen auszubauen, um die Abhängigkeit von anderen Akteuren zu verringern. Dazu gehören die schon erwähnte Schaffung einer eigenen städtischen Bank, die das System der autorisierten Banken faktisch ablöst, Tendenzen der Remunizipialisierung von Unternehmen und im Juni 1997 auch die Schaffung eines eigenen landesweiten Fernsehzentrums, obwohl mit NTV (und dem MOST-Medienkonzern insgesamt) einer der Verbündeten Luschkovs, Vladimir Gussinskij, über die einflußreichste private Medienunternehmung verfügt. Die Moskauer Regierung hält 67\% der Aktien des neuen Fernsehzentrums. Be- 
obachter sehen darin auch den Versuch Luschkovs, sich eine eigene rußlandweite öffentliche Plattform zu verschaffen, die in deutlicher Opposition zu den »Reformern « in der Regierung steht und für populistische und nationalistische Positionen offen ist, die innerhalb der MOST-Gruppe auf Widerstand stoßen (Fossato/Kachkaeva 1997).

\section{Die Rolle organisierter Kriminalität und Korruption}

Eine der kompliziertesten Fragen ist die nach der Stellung der organisierten Kriminalität innerhalb des politisch-wirtschaftlichen Systems Moskaus und von noch größerer Bedeutung - nach der Nutzung von extralegalen und illegalen Methoden durch die Moskauer Regierung und die mit ihr verbündeten Unternehmensgruppen. Eine Diskussion dieser Probleme erweist sich schon deshalb als schwierig, weil in der breiten Öffentlichkeit faktisch kein Unterschied zwischen legaler und illegaler Machtausübung gemacht wird. Meinungsumfragen weisen aus, daß die Moskauer Bevölkerung die von ihr später mit überwältigender Mehrheit gewählten Repräsentanten (die Moskauer Regierung) mit »mafiaähnlichen Gruppierungen« gleichsetzen (Muštuk 1995: 25-26; Levinson 1994: 245).

Eine zweite Schwierigkeit besteht im Fehlen verläßlicher Information. Das bekannteste seriöse Buch über das organisierte Verbrechen Moskaus, das eine Analyse der Entstehung, der Strukturen und Aktionen verschiedenster krimineller Gruppen und ihrer Paten vorlegt (Modestov 1996), geht in keiner Weise auf irgendwelche Beziehungen zwischen diesem »Untergrund « und der Moskauer Regienung ein. Obwohl das Buch an einigen Stellen Fakten erwähnt, die auf Beziehungen krimineller Gruppen mit Vertretern der föderalen Regierung, der Russischen Duma und der Administration des Präsidenten sowie russischen Sicherheitsdiensten hinweisen, werden Kontakte zu Beamten der Moskauer Regierung völlig ausgeklammert. Timothy Colton streift das Problem auf nur zwei Seiten von 200, die er Moskaus Entwicklung nach 1985 widmet. Auch seine Darstellung bleibt bei der Wiedergabe von Gerüchten und dem Verweis auf einige wenige Einzelfälle stehen (Colton 1995: 742-744). Die Quellenlage erlaubt es nicht, über diese Darstellungen hinauszugehen.

Es ist bekannt, daß Popov, der erste frei gewählte Bürgermeister Moskaus, während seiner Amtszeit zu einem reichen Geschäftsmann wurde. Eine ganze Reihe von Beamten verkoppelten dienstliche und private Interessen bis zur Ununterscheidbarkeit. 1992 realisierte Luschkov die Idee, Beamte dadurch zu stimulieren, daß ihnen ein Anteil an dem durch sie getätigten Vertrag zwischen der Stadt und dem privaten Investor zukommt. Als die Öffentlichkeit eine Überprüfung dieser Praktiken verlangte, verwies Lužkov auf die Gerichte, die allein Korruptionsvorwürfe zu untersuchen hätten 
(Argumenty i fakty, Nr. 41, Oktober 1992). Die folgende Nachricht, publiziert in Moskovskij Komsomolec, ist ein Beispiel für die Verquickung legaler und extralegaler Formen:

»Der Bürgermeister von Moskau, Jurij Luschkov, erhielt von seinen eigenen Untergebenen ein nettes Geschenk. Wie MK (Moskovskij Komsomolec - M. B.) informiert wurde, hat die Moskauer Regierung beschlossen, Luschkov für seinen wertvollen Beitrag zur Organisation und Realisation von städtischen und föderalen Programm des Jahres 1996 mit einer Prämie in Höhe eines sechsmonatigen Gehaltes auszuzeichnen. (Moskovskij Komsomolec, 21. Februar 1997)

Moskau erscheint als diejenige Großstadt Rußlands, die am meisten kriminalisiert und durch Korruption geprägt ist. Einige Experten gehen davon aus, daß es in der Stadt rd. 200 kriminelle Gruppen gibt, die über 2.000 aktive Gangmitglieder und 6.000 unterstützende Personen verfügen (Gurov 1995: 312). In anderen Quellen wird davon ausgegangen, daß die größte kriminelle Gruppe Moskaus für sich genommen schon 2.000 Mitglieder habe (Kryštanovskaja 1995: 98). Trotzdem ist die Lage nicht völlig außer Kontrolle. Die starken politischen und wirtschaftlichen Gruppen sind in der Lage, das Verhalten der kriminellen Gruppen in einem bestimmten Maße zu beeinflussen sei es durch Kooperation, sei es durch Einschränkung ihres Wirkungsfeldes (z.B. im Zentrum Moskaus - Modestov 1995: 191) oder sogar durch ein gemeinsames Vorgehen mit einigen dieser Gruppen gegen andere (so 1993/94, als die Moskauer Polizei, private Sicherheitskräfte und russische kriminelle Gruppen gemeinsam Gruppen der »tschetschenischen Mafia« zurückzudrängen suchten). Zwischen den legalen und illegalen Akteuren herrschen zugleich symbiotische und Konkurrenzbeziehungen. Auch die Bevölkerung sieht die Prosperität Moskaus mit dem mafiösen Charakter der Wirtschaft, den Schutzgelderpressungen, den vertraulichen Beziehungen zwischen den Führem legaler und illegaler Organisationen unlösbar verknüpft.

Vor dem Hintergrund dieser Beobachtungen scheint es offensichtlich, daß der Kampf gegen Korruption und organisierte Kriminalität nicht im Zentrum der politischen Aktivität der Moskauer Regierung steht, auch wenn offizielle Verlautbarungen anderes bekunden. Schreibt man dies nicht der »moralischen Verderbtheit« der Politiker zu und geht davon aus, daß sie sich vor allem als erfolgreiche Politiker zu behaupten suchen, dann stellt sich die Frage, unter welchen Bedingungen es für einen Politiker rational sein könnte, extra- oder auch illegale Praktiken zu tolerieren oder sogar zu unterstützen.

Die einzige Antwort, die hier gegeben werden kann, ist rein hypothetisch. Unter den russischen Bedingungen ist der Kampf gegen Kriminalität und Korruption besonders aufwendig und würde hohe Kosten verursachen. Der Markt entstand in der Sowjetunion der siebziger Jahre als Schwarzmarkt. Er bildete sich unter der Schirmherrschaft der organisierten Kriminalität heraus. Die Welle der Gründung von legalen Kooperativen und privaten Unternehmen ging Hand in Hand mit Bestrebungen der Nomenklatura, das staatliche 
Eigentum zu eigenen Gunsten zu privatisieren, und diente zugleich der Legalisierung des Schwarzmarktes. Die gesamte private russische Wirtschaft und große Teile der staatlichen Wirtschaft sind in ihrem Kern durch die Verbindung legaler, halblegaler und illegaler Praktiken gekennzeichnet und bisher auch nur in dieser Gestalt funktionsfähig. Die Kooperation mit kriminellen Gruppen beruht zumeist auf wechselseitigen Interessen und ist angesichts des weitgehenden Zusammenbruchs des staatlichen Gewaltmonopols auch unverzichtbar. Nachfrage und Angebot solcher "Dienstleistungen« (Gambetta 1988) sind vorhanden. Ein ernsthafter Kampf gegen Kriminalität und Korruption müßte es mit fast allen wichtigen wirtschaftlichen und politi= schen Interessengruppen und ihrer Wirtschaftsweise aufnehmen und hätte zugleich mit dem offenen Terror krimineller Gruppen zu rechnen. Hinzu kommt, daß die Kriminalisierung der Wirtschaft zumindest teilweise auch ein Stück Unabhängigkeit von zu starker Politisierung ökonomischer Prozesse zu ermöglichen scheint (vgl. dazu Pejovich 1997: 22).

Diesen absehbar hohen Kosten des Versuchs der »Reinigung《 der Wirtschaft und Politik von Kriminalität und Korruption stehen keinerlei entsprechend hohe Aussichten auf rasche Gewinne in diesem Kampf gegenüber. Dem Verweis auf das Kollektivgut einer »sauberen« Wirtschaft und Politik, die mittelfristig auch höheres Wachstum und Wohlstand sowie persönliche Sicherheit garantieren würden, steht der Umstand gegenüber, daß zumindest kurzfristig Politiker und Unternehmer aus der gegebenen Situation durchaus große Gewinne ziehen konnten. Ein Arrangement mit den kriminellen und korruptiven Tendenzen und ihrer Nutzung für die eigenen Ziele scheint vielen Politikern unter dem aktuellen Kosten-Nutzen-Aspekt wesentlich günstiger als der Versuch, diese um jeden Preis zu bekämpfen. So scheint die Moskauer Regierung einige informelle Absprachen getroffen zu haben, um die Aktivität der kriminellen Gruppen in bestimmten Bereichen einzuschränken und ihnen dafür Duldung zugesichert zu haben. Im Austausch für den privilegierten Zugang einiger Unternehmergruppen zu staatlichen Ressourcen haben sich diese an der Finanzierung öffentlicher Ausgaben beteiligt und die Regierung unterstützt. Die eigenen Seilschaften wurden durch Korruption fest an den obersten Patron, den Moskauer Bürgermeister, gebunden, ohne dessen Schirmherrschaft sie gerichtlich verfolgt werden würden. ${ }^{5}$ Die Kosten für die Herstellung einer von Kriminalität und Korruption weitgehend freien Wirtschaft und Politik wurden »eingespart« und zugleich wurden zusätzliche Ressourcen für den Erhalt des politischen Regimes der Stadt freigesetzt. Solange es keine alternativen

5 Es ist charakteristisch, daß dem Verlust des öffentlichen Amtes in Rußland oftmals eine gerichtliche Untersuchung über Korruption und anderen Amtsmißbrauch folgt, während aktuelle Amtsinhaber eine weitgehende Freiheit von ernsthaften gerichtlichen Verfolgungen genießen. 
Akteure gibt, die erfolgreich eine andere Kosten-Nutzen-Rechnung präsentieren können, werden die wichtigsten politischen und wirtschaftlichen Gruppen und die Bevölkerung Moskaus eine solche Politik sehenden Auges unterstützen. Sie werden die herrschende Elite als Mafia titulieren und sie mit großer Mehrheit demokratisch wählen.

\section{Moskaus politisches Regime und seine Perspektiven aus der Sicht des Konzepts »urbaner Maschinen«}

Nach einer Periode der Wirren scheint Moskau einen Zustand soziopolitischer Stabilität erreicht zu haben. Seit mehr als sechs Jahren wird es von einer demokratisch gewählten Verwaltung regiert. Die Symbiose von Herrschaft einer Staatsapparatepartei unter Führung eines charismatischen Politikers mit der Formierung eines munizipalen Kapitalismus, der seine relative Prosperität vor allem der Konzentration der Revenue der russischen Umbruchsprozesse in der Hauptstadt verdankt, hat sich bis heute als lebensfähig erwiesen. Solche Entwicklungen werden in der jüngsten Literatur zur Transformationsforschung als Tendenzen der Herausbildung einer »delegativen Demokratie« bezeichnet, wo der gewählte Chef der Exekutive autorisiert ist, zu herrschen, " as he or she sees fit, constrained only by the hard facts of existing power relations and by a constitutionally limited term of office« (O'Donnell 1994: 59). Der geringe Grad an formeller Institutionalisierung und Gewaltenteilung sowie Rechtsstaatlichkeit wird durch starke informelle vertikale und horizontale Netzwerke und ein System persönlicher Herrschaft substituiert (O'Donnell 1996: 35) ${ }^{6}$. Dies wird mit »funktionalen Notwendigkeiten« und dem Ruf nach Massenpatronage in einer Übergangszeit gerechtfertigt.

Derartige Entwicklungen sind weder auf nationaler noch auf lokaler Ebene neu. Sie stellen offenbar mögliche Formen politischer Regime dar, die unter den Bedingungen des allgemeinen Wahlrechts und einer auf keinen gemeinsamen Nenner zu bringenden Überschneidung sozialer Faktoren eines schnellen sozialen Wandels auftreten können. Sie wurden zunächst in den USA im ausgehenden 19. Jahrhundert beobachtet und unter dem Begriff von machine politics zusammengefaßt. ${ }^{7}$ Die weite Verbreitung dieses

6 Eine breitere Auseinandersetzung mit diesen Problemen findet sich in Brie (1996a, 1996b, 1996c). Vgl. auch Gel'man (1996) und Urban (1997: 291f).

7 Frühe Analysen solcher machine politics finden sich bei Ostrogorski (1902 und 1910), Low (1904) sowie Hobson (1909). Max Weber hat diese Tendenzen früh rezipiert (1976: 842ff). Spätere Ausprägungen solcher politischen Maschinen sind u.a. dargestellt durch Scott (1972), Dix (1967), Legg (1969) und Weiner (1967). Bezogen auf die USA vgl. vor allem Dahl (1961), Banfield/Wilson (1963), Cornwell (1964), Merton (1984), Shefter (1976), Erie (1988) sowie die Sammelbände von Brownell/Stickle (1973) und Stave et al. (1984). Der italienische Fall wurde u.a. in den Arbeiten von Chubb $(1981 ; 1982)$ untersucht. 
Typs politischer Regime, seine sehr unterschiedliche Ausprägung und die völlig gegensätzlichen Folgen lassen die Frage offen, ob es sich dabei im jeweiligen Fall um ein Durchgangsstadium einer dynamischen Modernisierung oder um den Weg in die Sackgasse dauerhafter Stagnation handelt. New York und Palermo stehen für die gegensätzlichen Pole dieser Entwicklung urbaner politischer Maschinen.

Unter urbanen politischen Maschinen werden Organisationen verstanden, die in der Lage sind, die Input- und Output-Dimension des lokalen politischen Systems relativ dauerhaft zu kontrollieren (Erie 1988: 26). Gelingt ihnen das, so können sie über mehrere Wahlperioden in Folge die Exekutive besetzen und weitgehend unkontrolliert über die öffentlichen Ressourcen verfügen. Es entsteht ein sich selbst stützender Zyklus von gewonnenen Wahlen und darauf folgender Nutzung der öffentlichen Haushalte für die Formierung einer hinreichenden (minimalen) neuen Gewinnerkoalition. Ungeachtet der garantierten freien Wahlen ist der Wettbewerb rivalisierender politischer Gruppen um die Besetzung der Exekutive durch die monopolistische Ausbeutung der öffentlichen lokalen Ressourcen von seiten der regierenden Gruppe überlagert. Politische und wirtschaftliche Transaktionen sind untrennbar verbunden.

Die Macht urbaner Maschinen liegt in ihrer Fähigkeit, größere Teile der Wahlbevölkerung in Gruppen öffentlicher Massenpatronage zu verwandeln und einen klientelistischen Block mit wichtigen Gruppen der lokalen Wirtschaftselite zu bilden. Das allgemeine Wahlrecht wird nicht als System der Wahl von Repräsentanten auf der Grundlage eines öffentlichen Guts und entsprechender programmatischer Konfliktlinien genutzt, sondern als ein Mechanismus, einen Patron an die Macht zu bringen oder an der Macht zu halten, der sich der partikularistischen Interessen einer fragmentierten und abhängigen Wählerschaft annimmt und sie mit konkreten materiellen Gütern versorgt sowie symbolisch aufwertet. Der öffentliche Haushalt wird ausgegeben, um entscheidende Unternehmergruppen zu binden, das Schicksal von Kerngruppen der urbanen Maschine (ihre Funktionäre und jene, die eine Broker-Rolle bei der Gewinnung von Wählern spielen) unlösbar mit dem der Organisation zu verkoppeln, und um einer hinreichenden Zahl von Wähler-

8 »The fate of the Mezzogiomo can be taken as a lesson for the Third World today, and the former Communist lands of Eurasia tomorrow, moving uncertainly toward selfgovernment. The 'always defect' social equilibrium may represent the future of much of the world where social capital is limited or nonexistent... Many of the formerly Communist societies had weak civic traditions before the advent of Communism and totalitarian rule abused even that limited stock of social capital. Without norms of reciprocity and networks of civic engagement, the Hobbesian outcome of the Mezzogiomo - amoral familism, clientelism, lawlessness, ineffective government, and economic stagnation - seems likelier than successful democratization and economic development. Palermo may represent the future of Moscow.« (Putnam 1993; 183) 
gruppen weniger kostspielige Güter zukommen zu lassen. Urbane Maschinen haben unabhängig voneinander und unter sehr divergierenden Bedingungen eine Reihe gemeinsamer Strategien entwickelt wie eine zentralistische Organisation, die Personalisierung der Macht (die Rolle des Bosses) und die Konzentration auf informelle gegenüber formellen Interaktionsformen.

Lokale politische Regime, die durch urbane Maschinen beherrscht werden, schließen politische und wirtschaftliche Akteure auf der nationalen wie der lokalen Ebene ein. Sie regulieren den Austausch zwischen nationalen Eliten, der urbanen Maschine und ihrem Boß, den Funktionären und öffentlichen Angestellten, der Wahlbevölkerung und ihren verschiedenen Gruppen, wichtigen Teilen des Unternehmertums und krimineller Gruppen. Es wird ein Netzwerk wechselseitiger Abhängigkeit geschaffen, daß auf mehr oder minder stillschweigenden Vereinbarungen beruht, und von keinem der Teilnehmer verlassen werden kann, ohne dabei den Zugang zu wichtigen Ressourcen zu verlieren (Tabelle 1). Solche Regime schließen eine starke Legislative und rechtsstaatliche Judikative aus.

Die Herausbildung von Regimen, die durch die Dominanz urbaner politischer Maschinen gekennzeichnet sind, hängt von drei Variablen ab: (1) Sie können dann entstehen, wenn größere Teile der Bevölkerung und einige wichtige lokale Wirtschaftsgruppen von öffentlicher Unterstützung abhängig sind und Patronage erwarten. Eine von staatlicher Patronage abhängige $\mathrm{Be}-$ völkerung (einschließlich eines größeren Segments abhängigen Unternehmertums) ist die wichtigste soziale Voraussetzung für die Entstehung urbaner Maschinen. (2) Es werden genau dann Anstrengungen zum Aufbau solcher politischer Maschinen unternommen, wenn die lokalen Eliten von der Wahlunterstützung durch die Bevölkerung abhängig sind. ${ }^{9}$ Das allgemeine Wahlrecht auf lokaler Ebene ist die wichtigste politische Voraussetzung für derartige Regime. (3) Die beiden genannten Voraussetzungen gegeben, entstehen urbane Maschinen unvermeidlich, wenn es lokalen politischen Eliten in Kooperation mit nationalen Eliten gelingt, die notwendigen rechtlichen Rahmenbedingungen zu schaffen und die erforderlichen Güter für Massenpatronage bereitzustellen. Eine von staatlicher Massenpatronage abhängige Bevölkerung, lokales Wahlrecht und Kooperation lokaler und nationaler Eliten stellten jede für sich eine notwendige Bedingungen der Vorherrschaft urbaner Maschinen dar. Nur in ihrer Gesamtheit sind sie aber hinreichend für deren Durchsetzung. Eine Bevölkerung, die ihre demokratisch abgegebene Wahlstimme gegen Massenpatronage austauscht, weil sie keine besseren Alternativen sieht - dies ist die Grundlage urbaner Maschinen.

9 iln one sense, popular elections can be seen as a reestablishment of the redistributive mechanisms of the traditional setting. Once again a patron's position becomes somewhat more dependent on the social approval of his communiry ... « (Scott 1977: 140). 
Tabelle 1: $\quad$ Modell der Austauschbeziehungen zwischen Akteuren innerhalb eines politischen Regimes unter der Herrschaft einer urbanen Maschine

\begin{tabular}{|c|c|c|c|c|c|c|c|}
\hline $\begin{array}{l}\text { Nachirage } \\
\text { Angebot }\end{array}$ & $\begin{array}{l}\text { Nationale } \\
\text { politische Eliten }\end{array}$ & Der Boll & $\begin{array}{l}\text { Funktionüre/ } \\
\text { Öfentliche } \\
\text { Angestellte }\end{array}$ & Wählerschaft & Großunterme himer & $\begin{array}{l}\text { Kleinumler- } \\
\text { relomer }\end{array}$ & $\begin{array}{l}\text { kriminelle } \\
\text { Grupper }\end{array}$ \\
\hline $\begin{array}{l}\text { Nationale politische } \\
\text { Eliten }\end{array}$ & - & $\begin{array}{l}\text { Gesetze, nationale } \\
\text { Ressourcen, persönliche } \\
\text { Patronage }\end{array}$ & - & $\ldots$ & - & - & $\cdots$ \\
\hline Der Boß & $\begin{array}{l}\text { Unterstitzung } \\
\text { durch die Region } \\
\text { und persönliche } \\
\text { Unterstützung }\end{array}$ & - & $\begin{array}{l}\text { privilegierte } \\
\text { Jobs, privater } \\
\text { Zugang zu } \\
\text { offentlichen } \\
\text { Gütern } \\
\end{array}$ & $\begin{array}{l}\text { Massenpatro- } \\
\text { nage ausge- } \\
\text { wählter } \\
\text { Gruppen }\end{array}$ & $\begin{array}{l}\text { informeller Einfluß } \\
\text { auf öfentliche } \\
\text { Entscheidungen, } \\
\text { öffentliche Aufträge } \\
\text { und Konzessionen }\end{array}$ & $\begin{array}{l}\text { Massen- } \\
\text { patronage } \\
\text { ausgewähiter } \\
\text { Gruppen }\end{array}$ & $\begin{array}{l}\text { Tolerierung, } \\
\text { Protektion } \\
\text { gegen } \\
\text { staatliche } \\
\text { Verfolgung } \\
\end{array}$ \\
\hline $\begin{array}{l}\text { Funktionärel } \\
\text { Örentliche } \\
\text { Angestellte }\end{array}$ & - & $\begin{array}{l}\text { Loyalität, aktive Wahl- } \\
\text { unterstützung }\end{array}$ & - & $\begin{array}{l}\text { lokale } \\
\text { Patronage }\end{array}$ & persönliche Hilfe & $\begin{array}{l}\text { persönliche } \\
\text { Patronage }\end{array}$ & Kooperation \\
\hline Ẅ̈hlerschaft & - & $\begin{array}{l}\text { passive Wahl- } \\
\text { unterstützung }\end{array}$ & $\begin{array}{l}\text { klientelistische } \\
\text { Unterstützung }\end{array}$ & - & - & - & - \\
\hline Großnnternehmer & $\cdots$ & $\begin{array}{l}\text { Loyalität, persönliche } \\
\text { Unterstützung, } \\
\text { Bestechung }\end{array}$ & Bestechung & - & - & - & - \\
\hline Kleinunternehmer & - & Wahlunterstuitzung & Bestechung & - & $=$ & - & - \\
\hline $\begin{array}{l}\text { kriminelle } \\
\text { Gruppen }\end{array}$ & - & $\begin{array}{l}\text { Loyalität, persönliche } \\
\text { Unterstützung, Beste- } \\
\text { chung, Gewalt gegen } \\
\text { alternative Kandidaten }\end{array}$ & Bestechung & - & 一 & 一 & - \\
\hline
\end{tabular}


Urbane Maschinen entstanden - ungeachtet der ihnen gemeinsamen Merkmale - in sehr verschiedenen historischen Situationen, sie stellten politische Reaktionen auf unterschiedliche Veränderungen dar und haben vor allem auch zu völlig divergenten Resultaten geführt. Dafür stehen besonders die unterschiedlichen Schicksale nordamerikanischer und süditalienischer Städte. In den USA hat sich die Herrschaft urbaner politischer Maschinen als Durchgangsstufe einer dynamischen Modernisierung erwiesen und wurde durch politische Regime abgelöst, die im höheren Maße als ihre Vorgänger durch Gewaltenteilung, Rechtsstaatlichkeit und Ausdifferenzierung von Wirtschaft und Politik gekennzeichnet sind. In Süditalien dagegen, zumindest bis zum Zusammenbruch des Nachkriegsparteiensystems Anfang der neunziger Jahre, wurde der Weg einer Modernisierung ohne Industrialisierung eingeschlagen und ein weitgehend stagnatives politisch-wirtschaftliches Regime stabilisiert. Weder kann also angenommen werden, daß urbane Maschinen mit ihren bekannten Begleiterscheinungen von persönlicher Herrschaft, politischer Massenpatronage, engster Verschmelzung politischer und wirtschaftlicher Macht, Korruption, Kriminalität usw. nichts als die unvermeidlichen Übergangserscheinungen einer Zwischenstufe erfolgreicher Modernisierung sind, noch kann behauptet werden, daß sie unvermeidlich in Stagnation und in die dauerhafte Blockierung dynamischer Wirtschaftsentwicklung und rechtsstaatlich geleiteter Demokratisierung münden müssen.

Ein an anderer Stelle vorgenommener Vergleich nordamerikanischer und süditalienischer lokaler politischer Regime unter den Bedingungen der Vorherrschaft urbaner Maschinen mit dem politischen Regime Moskaus macht deutlich (Brie 1997b), daß letzteres bisher in vieler Hinsicht weder dem Typ dynamischer Modernisierung noch dem eines stagnativen Modernisierungspfades zuzuordnen ist, sondern eine spezifische Zwischenform zu bilden scheint. Dies gilt vor allem für die politische Kultur, die institutionelle Gestalt, das Wirtschaftssystem und die Beziehung zwischen lokalen und nationalen Eliten sowie den historischen Kontext des politischen Wandels. Prognosen müssen dieser Ambivalenz Rechnung tragen. Im folgenden wird das Moskauer politische Regime unter dem Aspekt der drei genannten, für die Entstehung und Dauer urbaner Maschinen typischen Variablen betrachtet.

(1) Existenz einer von staatlicher Patronage abhängigen Bevölkerung und Unternehmerschaft: Moskaus Ökonomie ist sehr heterogen. Sie wird gebildet durch die einflußreichsten Finanzgruppen Rußlands, Holdings von Rußlands wichtigsten Industrien, einem prosperierenden Dienstleistungssektor für die wachsende Oberklasse, einer großen Zahl von Kleinunternehmen, die die besonderen Bedingungen Moskaus zu nutzen vermögen, und einer Mehrheit von Betrieben und öffentlichen Einrichtungen, die völlig von staatlichen Subventionen abhängig sind. Die Mehrheit der Bevölkerung ist 
im öffentlichen Sektor beschäftigt. Für die Großunternehmen ist die Moskauer Regierung nur ein Kooperationspartner neben anderen, wichtig vor allem für die Schaffung der Infrastruktur einer modernen Metropole. Zugleich hat sich ein Block zwischen der Moskauer Regierung und einigen Großunternehmen herausgebildet, der auf föderaler Ebene agiert.

Die Moskauer Regierung war in der Lage, das zentralisierte staatssozialistische System der Massenpatronage der Moskauer Bevölkerung in ein System zu überführen, das unter den Bedingungen marktwirtschaftlicher Reformen und des allgemeinen Wahlrechts funktioniert. Mittlerweile stellt das Ausmaß dieser Patronage (vor allem bei den öffentlichen Stützungen der Ausgaben für die Betriebskosten im Wohnungssektor) eine hohe Belastung der öffentlichen Haushalte dar und gerät in Widerspruch zu weiteren marktwirtschaftlichen Reformen und Infrastrukturmaßnahmen. Es gibt die Drohung, die föderalen Subventionen radikal zu senken. Gleichzeitig entwickeln die Großunternehmen (durchaus in Kontinuität zu sowjetischen Zeiten) eigene Patronagesysteme, die zwar die Aufwendungen für die Stadt senken, zugleich aber die Basis öffentlicher Patronage reduzieren.

Die Moskauer Bevölkerung ist weit mehr von öffentlicher Patronage abhängig und an diese gewöhnt als der Durchschnittsbürger nordamerikanischer Städte vor hundert Jahren, aber weniger an personalistische Systeme von Patronage gebunden als dies in Süditalien während der Nachkriegszeit der Fall war. Die Möglichkeiten, diese Patronage zu verlassen (ExitOption), sind geringer als in den nordamerikanischen Städten, aber größer als in Süditalien. Vor diesem Hintergrund gibt es zwei Möglichkeiten: Entweder kommt es zur Schließung des öffentlichen Patronagesystems unter der Kontrolle einer Koalition von korrupter Administration und Großunternehmern, die von den föderalen Eliten unterstützt wird. Dann könnte entweder die gegenwärtige Staatsapparatepartei unangefochten ihre Position behaupten oder käme - im Falle des eigenen Versagens - unter den Druck einer alternativen urbanen Maschine, die sie innerhalb der gegebenen Struktur zu verdrängen sucht. Oder aber es setzen sich Tendenzen der weiteren Öffnung dieses Systems durch, neue wirtschaftliche Möglichkeiten werden durch die dynamischsten sozialen Kräfte genutzt, um sich eine soziale Existenz unabhängig vom Patronagestaat aufzubauen. Dies würde die soziale Grundlage eines Regimes auflösen, wo eine Staatsapparatepartei in der Lage ist, sich durch Wahlen dauerhaft an der Macht zu halten.

(2) Die politische Landschaft Moskaus ist dadurch geprägt, daß die Regierung das Monopol auf politisches Handeln besitzt. Das frühere Zentrum kommunistischer Macht ist ohne starke kommunistische Opposition. Die anderen Parteien sind bisher weitgehend politische Klientelgruppen und können mit der politischen Maschine Luschkovs nicht ernsthaft konkurrie- 
ren. Das politische Regime Moskaus ist nicht um eine Partei bzw. ein Parteiensystem, sondern um die staatliche Exekutive zentriert.

Diese Usurpation der Kontrolle über das politische Leben durch die Spitze der Moskauer Exekutive reduziert die Abhängigkeit der Führungsgruppe von der Wählerschaft auf ein Minimum. Thre Fähigkeit, sich als unersetzbarer öffentlicher Patron zu präsentieren und jede Alternative zu blockieren, ist sehr hoch. Wie aber jedes andere Regime mit derartigen autoritären Merkmalen könnte es in Zeiten einer Krise selbst durch kleinere oppositionelle Gruppen verwundbar sein, da größere Gruppen der Bevölkerung sich diesen Gruppen in kurzer Zeit mehrheitlich zuwenden könnten, wenn die Regierung in ihrer Rolle als effizienter Patron versagen würden (Voice-Option).

Die Monopolisierung von Politik durch die staatliche Exekutive und die damit verbundene Reduktion des politischen Lebens auf staatliches Handeln untergräbt nolens volens die politische Machtbasis der Moskauer Regierung gegenüber dem föderalen Zentrum. In dem Maße, in dem die Abhängigkeit von der Bevölkerung sinkt und diese zu reinen Wahlbürgern und Klienten öffentlicher Patronage werden, sinkt auch die Bedeutung der demokratischen Legitimation, die die Wähler der eigenen städtischen Regierung erteilen. Diese Legitimation ist aber der wichtigste Teil der politischen Macht Luschkovs und seiner Regierung gegenüber den föderalen Eliten. Ohne die Möglichkeit der Moskauer Regierung, im Konfliktfall mit der politischen Mobilisierung der Bevölkerung drohen zu können, kann der russische Präsident unter bestimmten Bedingungen ohne größeres politisches Risiko das System der Einsetzung des Bürgermeisters in der Hauptstadt von oben wiederherstellen und/oder ihn in einen Strohmann verwandeln.

(3) Bisher sehen die föderalen Eliten ihr Interesse an der politischen Stabilität in der Hauptstadt erfüllt. Die während der Perestroika hochgradig mobilisierten Gruppen der sowjetischen Mittelklasse sind gebunden und die Bevölkerung unterstützt weitgehend den Kurs der städtischen Regierung. Differenzen dieser Regierung mit dem Kurs auf föderaler Ebene werden deshalb toleriert. In Zeiten politischer Krisen hat sich die Moskauer Regierung unter Luschkov immer wieder bedingungslos auf die Seite des Präsidenten gestellt. Sie hat es verstanden, die Auseinandersetzungen auf jene Bereiche zu beschränken, die den Grundkonsens der herrschenden russischen Elite nicht infrage stellen. Dies könnte sich dann ändern, wenn der Kampf um die Nachfolge Jelzins offen ausbricht und Luschkov als einer der mächtigsten Prätendenten antritt. Die Versuchung, ihn auszuschalten und seine Machtbasis, das besondere politische Regime Moskaus, zu beseitigen, wären sehr groß.

Das politische Regime Moskaus scheint stabil. Aber die sozialen und politischen Voraussetzungen und das notwendige $\mathrm{Maß}$ an föderaler Unterstützung 
sind gerade in Moskau weit schwächer als zumeist angenommen. Die Möglichkeit, daß Moskau in eine politisch-wirtschaftliche Domäne verwandelt wird, die direkt dem Präsidenten unterstellt ist, geführt durch einen Gouverneur, der von ihm eingesetzt wird oder bestätigt werden muß, ist hoch. Die direkte Involvierung der städtischen Moskauer Regierung in den föderalen Machtkampf weit über das Maß und die Bedeutung anderer regionaler Regierungen hinaus, die vergleichsweise hohen sozialen Exit-Optionen der Wählerschaft und die geringe politische Mobilisation machen die Moskauer Regierung und das von ihr kontrollierte Regime durch Attacken einer sich ihr gegenüber vereinigenden föderalen Elite relativ leicht verwundbar. Würde Luschkov selbst der nächste russische Präsident werden, so könnte er, um sich einen Rivalen vom Halse zu halten, selbst derjenige sein, der die Wahlen eines ihm genehmen Bürgermeisters Moskaus in die eigenen Hände nehmen oder das System von Direktwahlen in Moskau ganz abschaffen würde. Die Zukunft von Moskaus politischem Regime hängt von den Machtverhältnissen zwischen den Elitegruppen der russischen Föderation ab. Es könnte ihrem Kampf in relativ kurzer Zeit zum Opfer fallen.

$\mathrm{Zu}$ einer Zeit, wo das gegenwärtige politische Regime Moskaus möglicherweise den Zenit seiner Entwicklung erreicht hat, haben sich teils spontan und teils durch direkte Nachahmung und damit verbundener institutioneller Diffusion die in Moskau approbierten Muster in der Mehrheit der russischen Regionen durchgesetzt (Gel'man 1996). Ihre große Schwäche angesichts der geringen Ressourcenausstattung, einer tiefen wirtschaftlichen Krise und gravierender sozialer Probleme wird nur durch die fast unlösbaren Schwierigkeiten kompensiert, gegenwärtig politisch-soziale und ökonomische Alternativen zu einem System regionaler delegativer Demokratie zu entwickeln. Außerdem profitieren sie von der Politik der Zentralregierung, die an der Existenz abhängiger Regionen und ihrer Stabilisierung interessiert ist, diese über einzeln ausgehandelte Verträge an sich bindet (Kirkow 1997) und sich gegenüber der konkreten Art und Weise der Stabilisierung dieser Regionen weitgehend neutral verhält. Es ist eine Isomorphie von präsidialer Macht in Rußland und ihrer Stützung durch informelle gesamtrussische Interessengruppen einerseits und den regionalen Regimen von Gouverneursherrschaft und innerregionalen Allianzen entstanden. Vor diesem Hintergrund wäre es eine Ironie der Geschichte, wenn das Ende der lokalen delegativen Demokratie in Moskau mit der Stabilisierung dieses Regimetypus in den anderen russischen Regionen zusammenfallen würde.

\section{Literatur}

AEN (1994): Mer Moskvy: nalogovye služby rabotajut »udovletvoritel'no«. Moskovskie Novosti, 7. August, S. 27.

Artsruni, Vartkez (1996): »Gorod voshel' v do'ju«, Moskovskie Novosti, 14. Juli, S. 11. 
Babak, Elena (1993a): Merija pytaetsja saščitit' rjadovykh pokupatelej. Moskovskie Novosti, 8. August, S. 5.

- (1993b): Pravitelstvo Moskvy popytalos' razobratsja so stolichnymi SP. Moskovskie Novosti, 5. September, S. 1.

Banfield, Edward C./ Wilson, James Q. (1963): City Politics. New York: Vintage.

Baranov, Sergej D. (1996): Gruppy davlenija v sovremennoj Rossii. Unveröfi. Vortragsmanuskript.

Brie, Michael (1996a): Rußland: Das Entstehen einer »delegierten Demokratie«. In: Merkel, Wolfgang; Sandschneider, Eberhard; Segert, Dieter (Hg.): Systemwechsel 2. Die Institutionalisierung der Demokratie. Opladen: Leske + Budrich: 143-178.

- (1996b): Rußland: Die versteckten Rationalitäten anomisch-spontaner Wandlungsprozesse. In: Hedwig Rudolpf (Hg.) unter Mitwirkung von Dagmar Simon: Geplanter Wandel, ungeplante Wirkungen. Handlungslogiken und -ressourcen im Prozeß der Transformation. WZB-Jahrbuch 1995: 44 - 61.

- (1996c): Transformationsgesellschaften zwischen Institutionenbildung und Wandel des Informellen. Berlin 1996: MPG-Arbeitsgruppe Transformationsprozesse. Arbeitspapiere AG TRAP 96/8.

- (1997a): Moskau: Die Ideologie des Chozjajn. Zur politischen Kultur eines postsozialistischen urbanen Regimes. Ms. in Druck.

- (1997b): The Political Regime of Moscow - Creation of a New Urban Machine. Ms. in Druck.

Brownell, Blaine A./ Stickle, Warren E. (eds.) (1973): Bosses and Reformers. Urban Politics in America, 1880-1920. Boston: Houghton Mifflin Company.

Chinayeva, Elena (1996): Yurii Lushkov - The Man Who Runs Moscow. 1n: Transition, 2 (23 February), 30-33.

Chubb, Judith (1981): The Social Bases of an Urban Political Machine: The Case of Palermo. In: Political Science Quarterly, 96 (1) Spring, 107-125.

- (1982): Patronage, Power, and Poverty in Southern Italy. A tale of two cities. Cambridge: Cambridge University Press.

Colton, Timothy J. (1995): Moscow. Governing the Socialist Metropolis. Cambridge/ London: The Belknap Press of Harvard University Press.

Cornwell, Elmer E. (1964): Bosses, Machines, and Ethnic Groups. Annals 353 (May), 27-39.

Dahl, Robert A., 1961: Who Governs? Democracy and Power in an American City. New Haven: Yale University Press.

Davydova, Natal'ja (1991): Novoe pravitelstvo ne budet esdit' v ZlLach. Moskovskie Novosti, 7 July, S. 5.

- Zagrebnoi, Jurij (1996): Bolše, čem prosto mer. Moskovskie Novosti, 23. - 30. Juni, S. 9.

Dix, Robert H. (1967): Colombia: The Political Dimension of Change. New Haven: Yaale University Press.

Efremova, Inna (1993): Rossijskoi privatizacii mer Moskvy predpochel' germanskij menedžment. Moskovskie Novosti, 29. Dezember, S. 4.

Erie, Steven P. (1988): Rainbow's End. 1rish-Americans and the Dilemmas of Urban Machine Politics, 1840-1985. Berkeley/Los Angeles/London: University of California Press.

Fadin, Andrej (1996): Možet li Moskovskij mer pobedit' v presidentskoj gonke? Nezavisimaja gazeta, 24. Oktober.

Fossato, Floriano; Kachkaeva, Anna (1997): Russia to have new nationwide television network. RFL/RL Newsline 1 (36), 22. Mai.

Gambetta, Diego (1988): Fragments of an economic theory of the mafia. Archives Européennes de Sociologie, 29 (1), 127-145.

Gel'man, Vladimir (1996): Regional'nye režimy: zaveršenie transformacii? Svobodnaja mysl' (9), 13-21.

Gurov, Aleksandr (1995): Krasnaja Mafija. Moskva: Samosvet.

Hobson, John Atkinson (1909): The Crisis of Liberalism. London.

Ivanov, Viktor (1995): Moskva sdelala ešče odin šag k finansovoj samostojatel'nosti. Kommersant Daily, 16. März, S. 1-2.

Jasina, lrina (1994): Upolnomočennye banki sdelajut gorodskoj biudžet »prozračnym«. Moskovskie Novosti, 1.-8. Mai, S. 5.

Kasjanikova, Anastasija (1996): Svidanie ministrov na jachte. Moskovskij Komsomolec, 5. November, S. 1. 
Kirkow, Peter (1997): Im Labyrinth russischer Regionalpolitik: Ausgehandelter Föderalismus und institutionelle Veränderungen. In: Osteuropa, 47 (1), 38-51.

Kolosov, M. (1995): Partii v regionach: vlijanie i perspektivy. Vlast' (7), 22-38.

Kryštanovskaja, O(l'ga) V. (1995): Nelegal'nye struktury v Rossii. Socis (8), 94-106.

Legg, Keith R. (1969): Politics in Modern Greece. Stanford: Stanford University Press.

Lepechin, Vladimir (1995): Lobbizm. Moskva: Fond IQ.

Levinson, A.G. (1994): Termin »bjurokratija« v rossijskich kontekstach. Voprosy filosofii (78), 241-248.

Low, Sidney (1904): The Governance of England. London.

Lupkov, Jurij M. (1996a): My deti tvoi, Moskva. Moskva: Izdatelstvo »Vargius«.

- (1996b): Interview. Moskovskij Komsomolec, 8. Juni, S. 2..

- (1996c): Sevastopol' - rossijskij gorod na Krymskom poluostrove. Istorija s geografiej. Izvestija, 1. November, S. 2.

Melničenko, Sergei (1996): Zemlja i volja. Moskovskij Komsomolec, 8. Mai.

Merton, Robert K. (1984): The Latent Functions of the Machine. In: Stave, Bruce M./ Stave, Sondar Astor (eds.): Urban Bosses, Machines, and Progressive Reformers, 2nd Revised Edition, Malabar, Florida: Robert E. Krieger Publishing Company, 27-37.

Michajlov, Aleksandr (1996): Privatizatsija v Moskve okazalas' denežnym delom. Moskovskie Novosti, 2.-9. Juni, S. 21.

Modestov, Nikolaj (1997): Moskva Banditskaja. Dokumental'naja chronika kriminal'nogo bespredela 80-90-ch godov. Moskva: Zentrpoligraf.

Moiseev, Igor/Uchlin, Dmitrij (1994): Stolica - dlja slavjan? Moskovskie Novosti, 9.-16. Januar, S. 10.

Muštuk, Orest (1995): Moskovskie vlasti glazami "podvlastnych«. Biznes i politika (9), 25-31.

Nemcov, Boris, (1997): Razgovory o podkovernoj bor'be vice-premierov s premierom vymysel'. lzvestiia, 6 . Mai, S. 1.

O'Donnell, Guillermo (1994): Delegative Democracy. Journal of Democracy 5(1), 55-69.

- (1996): Illusions About Consolidation. Journal of Democracy 7 (2), 34-51.

Ostrogorski, Moise (1902): Democracy and the Organization of Political Parties. London/ New York.

- (1910): Democracy and the Party System in the United States. London/ New York.

Ožegov, S. I. (1987): Slovar' russkogo jazyka. Moskva: Russkii jazyk.

Pejovich, Svetozar, 1997: The Transition Process in an Arbitrary State: The Case for the Mafia. ib review, $1(1), 18-23$.

Presnjakova, Irina (1996): »Zolotoe« kol'tso stolitsy. Moskovskie Novosti, 1.-14. Januar, S. 16.

PriMERnaja programma (1996): Moskovskij Komsomolec, 17. Mai, S. 1-2.

Puškar, Dmitrij (1994): Seson ochoty na gostej. Moskovskie Novosti, 21. Februar, S. 4.

Putnam, Robert D. (1993): Making Democracy Work. Civic Traditions in Modern Italy. Princeton, N.J.: Princeton University Press.

Razuvaev, Vladimir V. (1996): Vremja udel'nych knjasej? Štrichi k potretu Jurija Lužkova. Svobodnaja mysl' (4), 39-5l.

Scott, James C. (1972): Comparative Political Comuption. Englewood Cliffs, N.J.: Prentice Hall. - (1977): Patron-Client Politics and Political Change in Southeast Asia. In: Schmidt, Steffe W./ Guasti, Laura/ Landé, Carl H./ Scott, James C. (eds.): Friends, Followers, and Factions. A Reader in Political Clientelism. Berkeley: University of California Press, 123-146.

Shefter, Martin (1976): The Emergence of the Political Machine: An Alternative View. In: Hawley, Willis D. et al. (eds.): Theoretical Perspectives on Urban Politics. Englewood Cliffs, N.J.: Prentice Hall, 14-44.

Stave, Bruce M./ Stave, Sondar Astor (eds.) (1984): Urban Bosses, Machines, and Progressive Reformers, 2nd Revised Edition, Malabar, Florida: Robert E. Krieger Publishing Company.

Sutela, Pekka, 1994: Insider Privatization in Russia: Speculations on Systemic Change. Europe-Asia Studies, 46 (3), 417-435.

Weber, Max (1976): Wirtschaft und Gesellschaft. Grundriß der verstehenden Soziologie. Fünfte, revidierte Auflage. Tübingen: J. C. B. Mohr (Paul Siebeck).

Weiner, Myron (1967): Party Building in a New Nation: The Indian National Congress. Chicago: The University of Chicago Press.

Želenin, Aleksandr (1997): Moskovskij mer i profsojuzy. Nezavissimaja gazeta, 8. Mai, S. 2. 